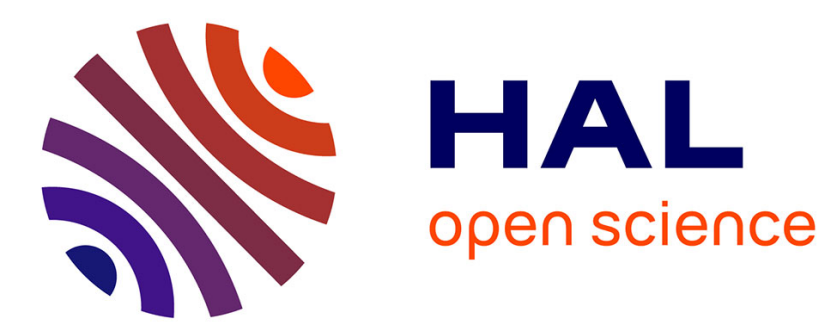

\title{
Vers une histoire de l'adoption internationale en France
} Yves Denéchère

\section{To cite this version:}

Yves Denéchère. Vers une histoire de l'adoption internationale en France. Vingtième siècle. Revue d'histoire, 2009, 102, pp.117-129. halshs-01091642

\section{HAL Id: halshs-01091642 \\ https://shs.hal.science/halshs-01091642}

Submitted on 5 Dec 2014

HAL is a multi-disciplinary open access archive for the deposit and dissemination of scientific research documents, whether they are published or not. The documents may come from teaching and research institutions in France or abroad, or from public or private research centers.
L'archive ouverte pluridisciplinaire HAL, est destinée au dépôt et à la diffusion de documents scientifiques de niveau recherche, publiés ou non, émanant des établissements d'enseignement et de recherche français ou étrangers, des laboratoires publics ou privés. 


\section{Vers une histoire de l'adoption internationale en France}

\section{Actualité et nécessité de l'histoire}

L'adoption d'un bébé né au Malawi par la chanteuse Madonna a défrayé la chronique en 2006-2007. De grands titres de la presse internationale se sont fait l'écho de la mobilisation des associations contre ce qui pouvait être perçu comme une mode pour vedettes riches. Audelà d'un épiphénomène médiatique "people », de réelles questions ont été posées sur la définition de l'orphelinat et de l'adoptabilité, la philosophie et la pratique de l'adoption internationale, le respect des législations nationales, les moyens d'assurer le bien-être d'enfants malheureux en les maintenant au sein de leur société, etc. ${ }^{1}$

En octobre 2007, Nicolas Sarkozy a chargé le journaliste Jean-Marie Colombani d'une mission sur l'adoption «en raison de son intérêt de longue date pour cette question et de son expérience personnelle». Celui-ci a déclaré vouloir œuvrer «avec l'espoir d'inverser les préjugés défavorables aujourd'hui à l'adoption internationale entre les soupçons de trafic et l'adoption hypermédiatisée de Johnny $»^{2}$. En effet, l'adoption d'une petite Vietnamienne par le couple Hallyday en 2004 a également suscité des polémiques lorsque les parents ont indiqué qu'une visite de Madame Bernadette Chirac au Vietnam avait permis de faciliter les choses $^{3}$. Au même moment des films de fiction voulaient mettre en lumière le «parcours du combattant» des candidats à l'adoption ${ }^{4}$. Quand il s'agit du «bonheur » d'un enfant, parfois même de sa survie, la fin ne justifie-t-elle pas les moyens? Les tribulations de l'Arche de Zoé au Tchad visant à l'expatriation d'enfants présentés comme orphelins, et la communication menée par cette association auprès de ses adhérents laissant entrevoir la possibilité d'adoptions ultérieures, ont mis en lumière des pratiques douteuses et la complexité de la donne politique.

Pourtant, depuis la convention internationale de La Haye du 29 mai 1993 (entrée en vigueur en France en 1998), les dérives sont rares. Le texte considère qu'une adoption internationale est celle qui implique le déplacement de l'enfant de son pays d'origine vers celui où vit sa famille adoptive (article 2-1). Selon cette convention, l'intérêt de l'enfant doit passer avant tout. Si possible, il vaut mieux qu'il soit adopté par des proches ou reste dans son pays d'origine. L'adoption dans les Etats étrangers signataires ne peut se faire que par l'intermédiaire d'un organisme agréé et toute forme d'achat des enfants ou de rémunération financière est bannie.

Ces éléments de définition et les épisodes évoqués plus haut mettent en lumière que l'adoption internationale doit être considérée comme une dimension des relations internationales. Le phénomène implique des Etats et leurs dirigeants, des associations et des filières d'adoption - les unes et les autres pouvant être tout à fait légales ou plus nébuleuses des particuliers, souvent des couples en mal d'enfants ou souhaitant mettre en œuvre une philosophie d'aide au développement ou du bien-être des enfants. Ceux-ci enfin, ne sont que des acteurs passifs des procédures de l'adoption internationale et des relations internationales en général. Cette passivité explique sans doute que jusqu'à une période récente les enfants ont été oubliés par l'historiographie des relations internationales. Seule la question des enfants en temps de guerre a été véritablement explorée : enfants victimes, enfants réfugiés, enfants

\footnotetext{
${ }^{1}$ Le Monde, «Adoption sans frontières à Hollywood»par Claudine Mulard, 2 novembre 2006, citant les quotidiens britanniques The Independent et Daily Express et australien Daily Telegraph.

${ }^{2}$ Le Monde, 4 octobre 2007 ; Le Figaro, 13 octobre 2007. Jean-Marie Colombani a présenté en mars 2008 les conclusions de son travail : Rapport sur l'adoption, La Documentation Française, 2008, 350 p.

${ }^{3}$ Libération, 17 novembre 2005, sur l'émission « En Aparté » de Canal +.

${ }^{4}$ Holy Lola, de Bertrand Tavernier, 2004 (un couple français désirant adopter une petite Cambodgienne) ; Nordeste, de Juan Solanas, 2004 (une Française cherchant à adopter un enfant argentin).
} 
soldats. Mais d'autres domaines peuvent faire l'objet d'études, l'adoption internationale est l'un d'entre eux. Porter un regard rétrospectif est indispensable pour éclairer l'actualité de ce phénomène, on peut en effet supposer que les dérives actuelles sont des survivances de pratiques antérieures mal codifiées.

La priorité est de mieux connaître les premières initiatives - très peu nombreuses dans l'entre-deux-guerres - qui après 1945 conduisent progressivement au développement de l'adoption internationale. Quels sont les types d'action entrepris ? Sous-tendus par quelles philosophies ? Répondant à quels contextes ? Depuis les années 1960, époque à partir de laquelle l'adoption internationale peut être envisagée comme un phénomène de société, on estime entre 300000 et 500000 le nombre d'enfants ayant fait l'objet d'une adoption internationale dans le monde. Pour la France, entre 1979 à 2007, le nombre est d'environ 80 000, ce qui la place au deuxième rang derrière les Etats-Unis ${ }^{5}$. Il faut trouver des explications à ce phénomène en interrogeant les rôles des différents protagonistes : candidats à l'adoption, intermédiaires, Etats, organisations internationales... Quelles sont leurs motivations ? Quelles relations entretiennent-ils entre eux ? En défendant quels points de vue ? L'histoire de l'adoption est à la fois une histoire du droit et une histoire des rapports entre enfants et adultes $^{6}$. Mais les adoptions transnationales sont à considérer comme de véritables mouvements internationaux Sud/Nord puis Est/Ouest (années 1990) vers la France. On conviendra qu'il faut ajouter l'histoire des relations internationales comme troisième approche nécessaire de l'adoption internationale puisque celle-ci est bien « une migration singulière ${ }^{7}$. Jusqu'à présent, cette dimension n'a été privilégiée.

\section{Les approches disciplinaires de l'adoption internationale}

La littérature sur l'adoption est pléthorique. Nombreux sont les témoignages de parents adoptants qui souhaitent faire partager leur expérience personnelle. Les titres très évocateurs de ces ouvrages montrent bien qu'il s'agit de récits de vie émouvants qui insistent sur les motivations des familles qui accueillent des enfants étrangers ${ }^{8}$. Tous soulignent peu ou prou le caractère laborieux de l'adoption internationale qui ajoute à la lourdeur de procédure nationale des difficultés supplémentaires liées à la législation internationale et à celles des pays offrant des enfants adoptables, sans oublier l'aspect financier des déplacements... Le parcours généralement décrit commence par la galère de l'attente, puis l'enthousiasme de l'accueil, la gestion des relations sociales, les réponses apportées aux questions identitaires de l'enfant, une adolescence parfois problématique... Presque toujours écrits lorsque l'enfant adopté a grandi, ces témoignages signalent la dimension psychologique d'une vraie aventure humaine qui évolue dans le temps. Bien que forcément plus récents mais désormais également nombreux, les récits d'enfants étrangers adoptés offrent un point de vue complémentaire?.

Sur le plan de la recherche universitaire, l'adoption est un sujet d'étude privilégié pour les juristes, dans plusieurs de leurs spécialités. Il est impossible de recenser tous les travaux

\footnotetext{
${ }^{5}$ Pour la France, les statistiques des ministères de la Justice, des Affaires sociales et des Affaires étrangères « ne se recoupent pas » selon Gérard Gouzes, Pour une éthique de l'adoption internationale, La Documentation française, 2000, p.19.

${ }^{6}$ Jean-Pierre Gutton, Histoire de l'adoption en France, Paris, 1993, Publisud, p.7.

${ }^{7}$ Brigitte Trillat, «Une migration singulière : l'adoption internationale », in L'adoption des enfants étrangers, séminaire Nathalie Masse 1992 du CIE, 1993, pp.15-25.

${ }^{8}$ Par exemples, Dominique Grange, Je t'ai trouvé au bout du monde, Paris, Stock, 1987 ; Maren Sell, La loi, c'est l'amour, Stock, 1997. Des récits sont publiés régulièrement dans Accueil, la revue de l'association Enfance et Familles d'Adoption (EFA) qui se définit comme « la seule revue spécialisée sur l'adoption » et dans la revue Accueillir éditée par le Service Social d'Aide aux Émigrants (SSAÉ) qui est une émanation du Service Social International (SSI).

${ }^{9}$ Revue Edelweiss, «Paroles d'adoptés », décembre 2003-janvier 2004. Barbara Monestier, Dis merci! Tu ne connais pas ta chance d'avoir été adoptée, Paris, Editions Anne Carrière, 2005.
} 
qui ont accompagné l'évolution de la législation sur l'adoption et qui accordent une place importante à l'adoption internationale ${ }^{10}$. Certaines publications portent exclusivement sur cette dernière dimension ${ }^{11}$. Toutes les sciences humaines et sociales ont des raisons de s'intéresser à l'adoption internationale. Marie-Pierre Marmier-Champenois s'est penchée sur la loi du 11 juillet 1966 (qui distingue adoption simple et adoption plénière) sous l'angle de la sociologie juridique ${ }^{12}$. Des psychologues ont été intéressés par le caractère interracial et transculturel de l'adoption et ses effets sur les enfants adoptés : «trouble de l'attachement», « souffrance du lien », etc. La récente thèse d'ethnologie d'Elise Prébin porte sur l'adoption internationale vue d'un pays de départ, la Corée du Sud. L'étude traite des séjours temporaires et très médiatisés d'adultes qui naguère ont été adoptés à l'étranger. La docteure, elle-même dans ce cas, a participé à un «retour en Corée » organisé par les autorités du pays ${ }^{13}$. Des médecins pédiatres travaillent également sur l'adoption comme le Dr Jean Vital de Monléon qui assure au CHU de Dijon, une consultation d'adoption outre-mer et est expert auprès du Conseil supérieur de l'adoption ${ }^{14}$.

En 1993, en concluant son Histoire de l'adoption en France, Jean-Pierre Gutton invitait les historiens à s'intéresser à cette question dont "les voies d'accès sont forcément multiples pour connaître un phénomène complexe ». En 1999, dans son livre L'adoption interraciale, Françoise Maury consacrait un chapitre à l'histoire de cette pratique, notamment l'adoption d'enfants coréens en France. Elle concluait : «l'adoption internationale et interraciale est un vaste champ où les recherches ne font que débuter ${ }^{15}$. Près de dix ans plus tard, on peut affirmer que les historiens français n'ont pas suivi quand certains de leurs collègues européens (suédois, néerlandais...) américains ou canadiens participaient aux réflexions sur les intercountry adoptions et les transracially adopted children ${ }^{16}$. Pour la France contemporaine, Ivan Jablonka a approché l'adoption en étudiant les enfants de l'assistance publique et le transfert d'enfants réunionnais en métropole. La chronologie, les caractères interraciaux et transculturels rapprochent l'exil des petits réunionnais de l'adoption internationale. En revanche, l'accueil de ces enfants a été très différent de celui reçu par les enfants étrangers adoptés - et attendus - en France ${ }^{17}$.

Si l'historiographie de l'adoption internationale est quasi inexistante c'est surtout en raison du caractère très récent de cette pratique. Les délais légaux régissant la consultation des archives publiques et le manque de recul sont les premiers éléments d'explication. C'est seulement maintenant que les archives s'ouvrent et permettent d'appréhender le phénomène. Cette histoire du temps présent ne peut commencer qu'aujourd'hui en croisant les sources, les méthodes et les concepts de l'histoire des relations internationales et de l'histoire sociale.

\footnotetext{
${ }^{10} \mathrm{Au}$ milieu de beaucoup d'autres : Brigitte Trillat, L'adoption. Essai sur les institutions, Lyon, PUL, 1995 ; François Boulanger, Enjeux et défis de l'adoption : étude comparative et internationale, Paris, Economica, 2001 ; Richard Crone, Mariel Revillard et Bertrand Gelot, L'adoption : aspects internes et internationaux, Paris, Defrénois, 2006.

${ }^{11}$ Jacques Foyer et Catherine Labrusse-Riou (dir.), L'adoption d'enfants étrangers, Paris, Economica, 1986 ; Isabelle Cogliati, «Adoption internationale et respect de l'enfant», Les Cahiers Rémois d'Etudes Internationales, 1999.

${ }^{12}$ Marie-Pierre Marmier-Champenois, L'adoption. Etude de sociologie juridique, La Documentation Française, collection ministère de la justice, 1969 ; L'adoption, Armand Colin, 1972 et autres éditions.

${ }^{13}$ Elise Prébin, Adoption internationale : les revenants de Corée, thèse en ethnologie, Paris X, 2006.

${ }^{14}$ Jean Vital de Monléon, Naître là-bas, grandir ici. L'adoption internationale, Paris, Belin, 2003.

15 Jean-Pierre Gutton, Histoire de l'adoption en France op. cit., pp.189-190. Françoise Maury, L'adoption Interraciale, Paris, L'Harmattan, 1999, p.304.

${ }^{16}$ Christina Zaar, Intercountry adoptions. What is the state of research and what new fields need to be investigated ?, Stockholm, Solna, 1991 ; Howard Altstein et Rita J. Simon (dir.), Intercountry Adoption : A Multinational Perspective, New York, Praeger, 1991, 204 p.

${ }^{17}$ Ivan Jablonka, Ni père, ni mère. Histoire des enfants de l'assistance publique (1874-1939), Paris, Le Seuil, 2006, 374 p. ; Enfants en exil. Transfert de pupilles réunionnais en métropole (1963-1982), Paris, Le Seuil, 2007.
} 


\section{Les matériaux à la disposition de l'historien}

Les archives du ministère des Affaires étrangères sont un point de départ obligé, celles des ministères de la Justice et des Affaires sociales sont moins accessibles et non exclusivement liées à la dimension internationale de l'adoption ${ }^{18}$. Bien qu'organisme interministériel, la Mission de l'Adoption Internationale dépend du Quai d'Orsay où elle a déposé un premier fonds d'archives. On y trouve surtout des documents datant des années 1980 et du début des années 1990, ce qui correspond aux premières années d'existence la MAI créée en $1988^{19}$. Pour la période antérieure, il faut chercher dans le fonds des affaires consulaires où quelques cartons sont très intéressants pour les années 1960 et $1970^{20}$. Dans ces deux fonds, les dossiers par pays sont particulièrement instructifs pour cerner les premiers mouvements d'adoption d'enfants étrangers en France et sur l'établissement de conventions bilatérales relatives à l'adoption comme la Convention franco-polonaise d'avril 1967 ou la Convention franco-yougoslave de mai 1971.

Les fonds des postes diplomatiques et consulaires français à l'étranger conservés au Centre des Archives diplomatiques de Nantes (CADN) recèlent des sources intéressantes mais dispersées et très rarement identifiées clairement comme relatives à l'adoption internationale $^{21}$. A Colmar, les Archives de l'occupation française en Allemagne conservent les dossiers des enfants nés de mères allemandes et de pères ressortissants français ou des nations alliées pour la période $1945-1952^{22}$. Une étude que nous avons réalisée montre que l'Etat français a pratiqué dans sa zone d'occupation une politique d'abandon/adoption dans un contexte certes exceptionnel mais en mettant en œuvre des mesures d'exception. Lorsqu'une mère désirait abandonner son enfant, elle le faisait en faveur des autorités françaises qui entreprenaient aussitôt des démarches pour son adoption en France. La récupération de ces enfants et la procédure de centaines d'adoptions (années 1946-1949) ont été unilatérales... l'Allemagne n'existant pas alors en tant qu'Etat. Il s'agit-là du premier mouvement d'adoption internationale de grande ampleur en France, très largement méconnu ${ }^{23}$.

Les associations qui ont participé activement à l'adoption internationale à partir des années 1960 et 1970, qui ont été agréées et/ou le sont aujourd'hui comme OAA (Organismes - anciennement (Euvres - Agréés pour l'Adoption), ont été contactées afin de répertorier leurs archives. L'association Les Amis des Enfants du Monde, créée en 1975 et qui a permis l'adoption de 7000 enfants étrangers en France dispose de peu d'archives ${ }^{24}$. L'association Solidarité Fraternité, créée en 1980 et qui a organisé l'adoption en France d'environ 600 enfants haïtiens, n'a que des archives très lacunaires pour les années $1980^{25}$. Cependant, ces associations conservent toujours sous une forme ou sous une autre leur histoire - au moins

\footnotetext{
${ }^{18}$ Des fonds d'archives de ces ministères peuvent être consultés au Centre des Archives Contemporaines de Fontainebleau. La sous-direction de la Famille de l'Enfance et de la Vie sociale a déposé des archives relatives à l'adoption internationale, mais les versements 19760172, 19760173, 19760175 et 19760185 étant entreposés dans un bâtiment amianté sont non consultables...

${ }^{19}$ Archives du Quai d'Orsay (AQO), MAI, versement des dossiers généraux (1972-1992), 23 cartons.

${ }^{20}$ AQO, Conventions administratives et Affaires consulaires, Affaires consulaires (CAAC) 1940-1978, cartons n॰3 à 6.

${ }^{21}$ CADN, Etat général des fonds et divers inventaires : quelques cartons bien identifiés dans le fonds Port-auPrince, des éléments plus dispersés dans les fonds Hanoi, Ho Chí Minh-Ville, Santo Domingo, Tananarive, etc.

${ }^{22}$ Bureau des Archives de l'occupation française en Allemagne et en Autriche (Colmar), répertoire numérique de la Direction des personnes déplacées, Enfants (5).

${ }^{23}$ Yves Denéchère, « Des adoptions d'Etat : les enfants de l'occupation française en Allemagne (1945-1952) » in Revue d'Histoire Moderne et Contemporaine, parution en 2009.

${ }^{24}$ Courriel du 28 février 2008.

${ }^{25}$ Entretien au siège de l'association à Renazé (53), 11 mars 2008.
} 
leur mémoire - afin de faire connaître aux candidats à l'adoption leurs valeurs ${ }^{26}$. De nombreuses associations et communautés religieuses sont moins faciles à joindre. Ce sont celles qui à un moment ou à un autre ont joué le rôle d'intermédiaire pour les parents s'engageant dans une démarche individuelle pour trouver un enfant à l'étranger. Il va sans dire que les conditions d'accès à leurs archives sont très aléatoires. La présidente d'une association m'écrit que « les archives sont comme l'histoire des villages africains des griots : tout dans la tête $» . .{ }^{27}$ Au niveau international, il faudrait regarder de près si les fonds du Service Social International et du Service Social d'Aide aux Émigrants (France) et de l'Union Internationale de Secours aux Enfants (liée à la Croix-Rouge) peuvent être utiles, notamment pour l'entre-deux-guerres. En revanche, il ne faut rien attendre de la Croix-Rouge française : les archives de son service "adoption », qui a fonctionné de 1941 à 1951, ont été détruites lors d'inondations dans les années $1960 \ldots$

Les sources imprimées sont aussi nombreuses que précieuses. Il faut penser aux rapports établis par des parlementaires, des organismes publics ou des associations qui s'égrènent depuis les années 1960 et dont la périodicité s'accélère dans les années 1990. Ils sont autant d'expertises rendues à un moment donné et permettent d'avoir une vision globale de l'évolution de l'adoption internationale ${ }^{28}$. La législation française sur l'adoption (1923, 1939, 1966, 1976 et 1996), ainsi que les textes internationaux relatifs aux droits des enfants $(1959,1965,1979,1986,1993 \ldots)$ sont aisément accessibles. Certains fonds documentaires sont particulièrement fournis comme celui du CIDEF (Centre International de l'Enfance et de la Famille), fermé en 1999, dont toute la documentation a été déposée à la bibliothèque universitaire d'Angers ${ }^{29}$. Des «modes d'emploi » ou « guides » de l'adoption internationale ont été rédigés par des associations, des juristes, des particuliers, etc. Très nombreux dans les années 1980 quand le phénomène de l'adoption internationale s'amplifie, ils sont vite dépassés en raison de la rapidité de l'évolution des législations et des politiques des Etats. Cependant, une étude systématique de ces guides aurait quelque intérêt pour l'historien.

Un dépouillement de la presse permettra de mesurer à quels moments et en posant quelles questions l'adoption internationale fait la une des journaux. De la prise de conscience tiers-mondiste des années $1960^{30}$ aux articles des années 1990 et 2000 dénonçant tous les maux de l'adoption internationale, il est possible de suivre le traitement médiatique de cette pratique. Les centaines de reportages consacrés aux orphelins du monde entier et à l'adoption dans les journaux télévisés depuis les années 1960, les dizaines de films documentaires réalisés sur ce thème ont eu un impact sur la représentation que les Français se sont faite de l'adoption pendant cette période. Tel reportage de Cinq Colonnes à la Une présentant le Noël 1960 des onze enfants d'origines diverses adoptés par Joséphine Baker a sans doute contribué au développement de ce que certains psychologues identifient comme le «syndrome Joséphine Baker ${ }^{31}$. En 1981, l'APAEC (Association des Parents Adoptifs d'Enfants Colombiens) est fondée «en réponse à un programme télévisuel donnant une image faussée

\footnotetext{
${ }^{26}$ En 1990, la Fédération des Rayons de Soleil de l'Enfance a publié : Historique des Rayons de Soleil, Paris. Les associations rappellent également régulièrement leur passé dans leurs bulletins ou revues.

${ }^{27}$ Courriel du 29 février 2008.

${ }^{28}$ Signalons particulièrement un rapport du SSAÉ de 1986 : Gokalp A. et Bertrand C., Rapport sur l'adoption internationale, 2 volumes ; les rapports des députés Jean-François Mattéi (1995) et Gérard Gouzes (2001).

${ }^{29}$ Le CIDEF a été créé en 1997 par la fusion du CIE (Centre International de l'Enfance, créé en 1949) et l'IDEF (Institut de l'Enfance et de la Famille, créé en 1984). Le fonds documentaire compte plus de 1000 titres de périodiques et plus de 20000 volumes, sans compter des dossiers géographiques ou thématiques dont plusieurs sur l'adoption internationale. http://bu.univ-angers.fr

${ }^{30}$ Par exemple les appels lancés en 1962 dans Le Canard Enchaîné par Valentine de Coin-Coin (alias le journaliste Pierre Chatelain-Taillade) en faveur des enfants du Tiers-Monde.

${ }^{31}$ INA, «Le Père Noël chez Joséphine Baker », Cinq Colonnes à la Une, ORTF, 6 janvier 1961, 08 min 12 sec. Selon Françoise Maury, le syndrome de Joséphine Baker (dont elle-même n'était pas atteinte) est le désir de se faire remarquer en adoptant un enfant étranger ( $L$ 'adoption Interraciale op. cit., pp.68-71).
} 
de l'adoption en Colombie $»^{32}$. Lorsqu'en 1990, dans l'émission Racines animée par Michel Polac, est diffusé un documentaire intitulé «Ces tout-petits venus d'ailleurs », combien de Français postulants à l'adoption se reconnaissent dans les cas présentés ? Quel impact a eu le documentaire d'Hubert Dubois, « Adoption : les dessous d'un trafic » ? $?^{33}$

L'ensemble de ces sources constitue un corpus suffisant pour permettre d'établir les faits dans leur chronologie, d'identifier des ruptures et des évolutions, de préciser les intentions et les actions des protagonistes de l'adoption internationale.

\section{Différentes logiques à l'œuvre}

Phénomène complexe, l'adoption internationale met en relation de nombreux acteurs. Postulants à l'adoption, associations, Etats, organisations internationales développent leur propre logique peu ou prou complémentaire ou conflictuelle avec celles des autres protagonistes. L'étude de ces différentes logiques, constamment en interaction et en évolution, semble une manière intéressante d'approcher historiquement l'adoption internationale en France.

\section{La logique de résultat des candidats à l'adoption}

Lorsque le nombre d'enfants français adoptables diminue fortement et que l'amélioration des moyens de communication le facilite, trouver un enfant à l'étranger devient pour les candidats à l'adoption un moyen d'arriver à ses fins. Mais avant ce tournant des années 1960 et 1970, il est nécessaire de définir les prodromes de l'adoption internationale (autour de la loi de 1923 ouvrant la voie à l'adoption de mineurs), et d'évaluer avec précision les effets des guerres du $\mathrm{XX}^{\mathrm{e}}$ sur la naissance du phénomène. Dès avant la fin de l'année 1936, des Français demandèrent à adopter des enfants espagnols réfugiés de la guerre civile. Mais les autorités comme les associations étaient toujours très méfiantes. L'UISE estimait que ceux qui étaient vraiment orphelins pourraient rentrer en Espagne lorsque la situation serait redevenue normale et n'envisageait pas l'adoption comme une solution. L'extrême prudence des autorités françaises et des organisations internationales était due à la difficulté de saisir les desseins réels des demandeurs ${ }^{34}$. Après la Seconde Guerre mondiale, l'adoption par des familles françaises d'enfants nés en Allemagne est une autre illustration de l'impact des guerres dans l'histoire de l'adoption internationale. Au cours des années 1960 et 1970, dans le flou juridique ambiant, des Français sont partis pour l'étranger, notamment au Vietnam en guerre, pour tenter de ramener un enfant. Au même moment arrivent en France des enfants boat people mais difficilement adoptables car sans papier... Il faudrait mieux cerner ces «pionniers de l'adoption internationale» (l'expression est de Françoise Maury), leurs moyens d'action, leurs attitudes vis-à-vis des autorités des pays d'origine, vis-à-vis de l'administration française.

On ne s'attardera pas ici sur les motivations personnelles des adoptants. Elles sont bien présentées dans plusieurs ouvrages qui différencient un avant et un après la loi de 1976 qui a permis l'adoption d'enfants en présence de descendants naturels et le développement de l'adoption pour raison humanitaire. On sait également que l'adoption internationale concerne bien plus les catégories socioprofessionnelles supérieures que les agriculteurs ou les

\footnotetext{
${ }^{32}$ Site Internet de l'APAEC $: \underline{\text { http://tchaucha.club.fr }}$

${ }^{33}$ INA, José Viera, «Ces tout-petits venus d'ailleurs », Racines, France 3, 22 juillet 1990. Sur le plateau Michel Polac reçoit Brian Bouillon-Baker pour évoquer sa vie au sein de la «tribu » de Joséphine Baker; Hubert Dubois, «Adoption : les dessous d'un trafic », Canal + / Planète, 57 min, 2004.

${ }^{34}$ Archives départementales de Maine-et-Loire, $13 \alpha$ 13, lettre de W. A. Mac Kenzie, secrétaire général de l'UISE, au préfet de Maine-et-Loire, 17 septembre 1936.
} 
ouvriers $^{35}$. En revanche, il est nécessaire de définir les raisons de leur «choix » du pays offrant des enfants à l'adoption. Le nombre de pays-sources des enfants adoptés en France a connu une augmentation importante. En 1974, les 12 \% d'enfants adoptés qui sont étrangers viennent surtout de Corée du sud, du Vietnam et d'Inde. En 1980, ils étaient originaires de 10 pays différents, 30 en 1990, 77 en 2004. En corollaire, le nombre de visas «adoption» délivrés par la France a explosé (935 en 1980, près de 3000 en 1990, plus de 4000 en 2004) ${ }^{36}$. Mais la question d'un "choix » éventuel a-t-elle quelque pertinence quand on évoque une logique de résultat? On constate que les Américains et les Français ont adopté beaucoup de petits Cambodgiens et Vietnamiens ; parce qu'ils nourrissent un sentiment de culpabilité lié aux guerres d'Indochine et du Vietnam ? Le choix de tel ou tel pays d'origine est-il dicté uniquement par la faisabilité, la moindre difficulté de mener à terme un projet long et onéreux ? Que penser de la part encore importante aujourd'hui (40\%), mais surtout pendant très longtemps prépondérante, des démarches individuelles d'adoption c'est-à-dire sans passer par un organisme agréé ? Jusqu'en 1985, l'agrément de la DDASS n'était pas obligatoire pour l'adoption d'un enfant étranger. Combien de postulants s'étant vus refuser un agrément pour l'adoption d'un enfant français (obligatoire à partir de 1967) ont-ils tenté leur chance à l'étranger, ont ramené en France des enfants pour les adopter?

Pour appréhender cette réalité, et alors que les archives des jugements d'adoption qui pourraient donner des indications ne sont pas ouvertes, sans doute conviendrait-il de réaliser une enquête d'envergure significative auprès de différentes générations d'adoptants en veillant à la diversité des origines des enfants qu'ils ont adoptés. Pour ce faire, l'aide des Associations de parents par pays d'origine (APPO) serait indispensable. Outre des questions ouvertes permettant aux personnes de répondre en utilisant l'angle qui leur paraîtrait le plus pertinent (et cela aurait déjà un intérêt), il faudrait au moins saisir les variables suivantes : connaissance de la langue et de la culture du pays d'origine, relations établies avec des missionnaires ou des coopérants, avec des orphelinats, séjour au moment de l'adoption, retour avec ou sans l'enfant par la suite, poursuite de relations avec ce pays, etc. Une telle enquête aurait des limites : par exemple, concernant des couples immigrés en France qui, stériles, ont fait le choix d'adopter des enfants de leur pays d'origine. Les enfants adoptés par des couples turcs, yougoslaves, polonais, vietnamiens sont alors entrés en France au titre du regroupement familial d'où la difficulté d'en connaître le nombre ${ }^{37}$.

Pour certaines célibataires, l'adoption internationale a pu être un moyen de contourner les dispositions défavorables qu'elles prêtaient aux DDASS sur l'adoption par des personnes seules. Même si des pays d'origine étaient hostiles aux postulants célibataires, l'aventure paraissait davantage possible. Des couples homosexuels ont pu entrer dans une logique de résultat qui s'est doublée d'une logique de transgression à l'égard des Etats-sources en leur cachant une donnée rédhibitoire ${ }^{38}$. L'organisation de la migration de femmes enceintes de pays-sources vers les pays d'accueil où elles accouchent est une autre forme de transgression. Comment cerner la participation plus ou moins passive et plus ou moins consciente des postulants à l'adoption à des «trafics d'enfants » dénoncés régulièrement par les Etats et les associations à travers la presse, mais dont la définition n'est pas aisée ? Il convient par exemple de distinguer le recours à l'argent, qui permet d'accélérer des procédures légales, et l'achat d'enfants aux origines mal définies. L'adoptabilité des enfants et le libre arbitre des parents biologiques sont les deux points névralgiques de toutes ces affaires qui se multiplient

\footnotetext{
${ }^{35}$ Notamment dans Françoise Maury, L'adoption interraciale op. cit. L'auteure consacre un chapitre éclairant aux motivations non spécifiques, non-recevables et recevables de l'adoption interraciale, pp.47-94. Voir également dans Mirentchu Galaïnena, L'adoption, voyage au bout d'un désir, Paris, La Découverte, 1988, chapitres 5 à 8 .

${ }^{36}$ Statistiques de la Mission de l'Adoption Internationale, Ministère des Affaires étrangères.

${ }^{37}$ Brigitte Trillat, «Une migration singulière... » in L'adoption des enfants étrangers op. cit., pp.15-25.

${ }^{38}$ Pour faire le point : Homoparentalités : état des lieux, (collectif), Eres, 2005.
} 
à partir des années 1980. Encore faut-il ajouter que la grande majorité des parents adoptifs de ces enfants ne se doutent pas de l'existence de tels crimes. Par ailleurs, les rumeurs sont légion mais les trafics véritablement organisés et prouvés semblent peu nombreux ${ }^{39}$.

Une fois l'adoption effective, la question des relations entre les adoptants et les pays d'origine est également à poser. De quelles manières sont suivies les évolutions, l'actualité de ces pays ? Les adoptants y sont-ils retournés, seuls ou avec l'enfant ? ${ }^{40}$ C'est la nature des liens entre des sociétés souvent très dissemblables qui est ici en jeu et leur poids dans les relations bilatérales entre les Etats. Lorsque les familles biologiques connaissent les familles adoptantes de leurs enfants, ce qui est fréquent dans des pays comme le Vietnam ou Haïti et dans les cas d'adoption «ouverte » (Polynésie française par exemple), quel type de contacts est entretenu, organisé, refusé ? Quelles réponses sont par exemple données à des demandes d'aide financière faites par la famille biologique ? Très souvent, les familles adoptantes se tournent vers les associations pour demander conseil.

\section{La logique d'action des associations}

Certaines associations ou organisations françaises qui ont structuré les relations entre les candidats à l'adoption et des Etats étrangers ont été créées dès l'entre-deux-guerres. Les plus nombreuses sont apparues lorsque l'adoption internationale est devenue plus ample. Pour étudier les unes comme les autres, la problématique essentielle porte sur leurs motivations et les types d'actions engagées. Dans les différentes périodes, de quelles manières se sont-elles imposées comme interlocuteurs et intermédiaires ? En pratiquant quelles formes de lobbying, en défendant quelle philosophie de l'aide à l'enfance, de l'assistance au développement?

L'histoire interne des associations est bien évidemment à prendre en compte, en particulier les variations qui ont accompagné leurs actions en matière d'adoption internationale. Le cas de Terre des Hommes est particulièrement intéressant. Après les débuts de l'association en Suisse (1960), Terre des Hommes-France fut créée en 1962. En 1965 et 1967 eurent lieu les premiers «accueils à vie» de trois enfants (un Algérien et deux Vietnamiens) et en 1969 TDH fut la première œuvre française agréée pour l'adoption d'enfants sud-coréens. Moins de dix ans plus tard, en 1978, l'ONG renonçait aux adoptions internationales et choisissait de privilégier d'autres formes d'action, surtout l'aide au développement dans les pays concernés ${ }^{41}$. Des associations humanitaires, religieuses, ou autres ont également organisé des parrainages plutôt que des adoptions. C'est le cas notamment de l'association Plan qui depuis 1937 a parrainé 1,3 million d'enfants dans plus de 40 pays. La question des motivations demeure : le parrainage d'enfants miséreux d'Haïti ou d'orphelins du SIDA au Burkina Faso ne procèdent pas des mêmes intentions que le parrainage de petits Palestiniens ou de petits Tibétains...

Pour d'autres associations, l'adoption en France demeure la meilleure action pour assurer l'avenir d'enfants du Tiers-Monde. Financer dans un pays étranger une structure d'accueil - dont la pérennité n'est jamais totalement garantie - pour 50 enfants sur une dizaine d'années est une charge considérable. Organiser l'adoption de (seulement) 20 enfants par an sur la même période, c'est sauver quatre fois plus d'enfants, définitivement... Telle est la logique d'action humanitaire qui motive de nombreuses associations créées dans les années

\footnotetext{
${ }^{39}$ Brigitte Trillat et Sylvia Nabinger, « Adoption internationale et trafics d'enfants : mythes et réalités », Revue Internationale de Police Criminelle, Interpol, n428, 1991, p.18-25.

${ }^{40}$ Entre autres témoignages de retour dans le pays d'origine : Christine Audefray, « Adoption. D'une histoire... à l'autre », Carnets du Viêt Nam, n¹7, janvier 2008, pp.9-11.

${ }^{41}$ CADN, fonds Port au Prince, carton 51, « historique de Terre des Hommes-France », en 1973, 239 enfants sont accueillis puis adoptés (147 viennent de Corée, 53 du Vietnam, 15 de Colombie, 14 d'Haïti, 10 du Bangladesh).; L'adoption dans tous ses états. Enjeux et pratiques, Fondation Terre des Hommes, 2004,112 p. ; Françoise Maury, L'adoption interraciale op. cit., pp.28-31 et 39.
} 
1980 et 1990. En même temps, elles apportent un service à des familles françaises désirant adopter; certaines ont été créées avant tout dans ce but. Une typologie est à faire sur l'ensemble de la période pour distinguer les OAA, reconnus comme intermédiaires par les Etats, et les associations favorisant les démarches individuelles des candidats français à l'adoption dans les pays étrangers. De quelle manière fonctionnent ces associations ? Qu'estce qui pousse une association à demander l'agrément du ministère des Affaires étrangères ou à ne pas chercher à l'obtenir?

Des Etats-sources ont exigé que les organisations étrangères - quelle que soit leur nature - travaillent avec des associations locales ou nationales reconnues par eux. Des conventions ont ainsi été rédigées précisant les motivations des unes et des autres. Dans ces cas, les associatifs français n'ont eu que peu de rapports avec les autorités administratives nationales (en Haïti par exemple). Dans le cas de la Corée du sud, la quasi-totalité des candidats français à l'adoption ont dû passer indirectement par l'organisation américaine Holt pour obtenir un enfant, ce qui est un cas de figure encore différent. Lors de la transition politique en Roumanie, en 18 mois (1990-1991) plus de 7000 enfants ont été adoptés par des étrangers, dans des conditions souvent inacceptables. L'ONG Médecins du Monde (créée en 1975) suspendit alors les activités de son département adoption, et joua un rôle d'expertise auprès des autorités roumaines lors de préparation de la loi sur l'adoption de juillet 1991 . Ensuite, le Comité National Roumain pour l'Adoption signa avec Médecins du Monde le premier protocole mis en application dans le cadre de cette nouvelle loi ; des relations de confiance réciproque s'étaient établies entre l'ONG et les responsables roumains ${ }^{42}$. Ce cas n'est pas courant. Les actions engagées par les associations françaises pour faire avancer les procédures d'adoption dans des Etats étrangers très sourcilleux du respect de leur souveraineté ont été parfois contre-productives et ont pu compliquer les relations entre Etats.

\section{La logique de régulation des Etats}

Si l'adoption internationale est avant tout une aventure individuelle s'appuyant souvent sur des intermédiaires, les Etats demeurent toujours responsables de la circulation des enfants. Il s'agit donc d'une dimension de la politique étrangère des Etats comme l'a montré le choix politique de la Chine d'offrir davantage d'enfants à certains pays d'accueil (Canada) et moins à d'autres (France). En même temps l'adoption internationale touche à une autre expression de la souveraineté la plus fondamentale des Etats avec la question de la nationalité. Le phénomène est donc forcément lié à leur évolution politique et juridique au $\mathrm{XX}^{\mathrm{e}}$ siècle, aux politiques migratoires, aux changements de mentalité des sociétés. Après la Seconde Guerre mondiale, en même temps que la France organise l'adoption d'enfants nés de pères français en Allemagne, elle refuse de voir des petits Français quitter le territoire national pour être adoptés par des Australiens ou des Américains ou même par des Français expatriés ${ }^{43}$. C'est le reflet de la politique populationniste menée en France au lendemain de la Seconde Guerre mondiale ${ }^{44}$. L'histoire des Etats et des relations internationales est une base indispensable pour comprendre les vagues successives de l'adoption internationale. Les adoptions de petits Coréens à partir des années 1950, de petits indochinois à partir des années 1960, de petits latino-américains dans les années 1970, de petits européens de l'Est dans les années 1990 ont tout à voir avec les bouleversements politiques et la misère des pays d'origine. Pour ce qui est de l'adoption internationale en France, le passé colonial français et

\footnotetext{
${ }^{42}$ Témoignage de Claude Hertz, responsable du département «adoption » à Médecins du Monde, in L'adoption des enfants étrangers... op. cit., pp.79-81.

${ }^{43} \mathrm{AQO}, \mathrm{CAAC}, \mathrm{n}^{\circ} 3$, correspondance entre le ministère de la Santé publique et de la Population et le ministère des Affaires étrangères (1946-1950).

${ }^{44}$ Voir Paul-André Rosental, L'intelligence démographique. Sciences et politiques des populations en France (1930-1960), Paris, Odile Jacob, 2003, notamment le chapitre 4.
} 
la sphère francophone sont à interroger. Par rapport aux autres principaux pays d'accueil, il existe une particularité française d'adoption d'enfants africains (Ethiopie surtout, Burkina Faso, Mali...) et de Madagascar. Le fait qu'un enfant provienne d'un pays où la langue française est connue est-il un élément déterminant. En 1989-1990 la France (re)découvre en même temps que les images des orphelinats roumains, un pays de culture et de langue romane, proche et pouvant offrir des enfants de type européen à l'adoption...

Les Etats d'accueil des enfants étrangers doivent assurer des tâches de régulation: contrôle, organisation, reconnaissance. Les autorités consulaires françaises n'ont jamais délivré de visas d'entrée en France sans vérifier les papiers des enfants. Depuis le début des années 1970, l'Etat français n'a eu de cesse de réguler l'adoption internationale. En juillet 1971, se réunit pour la première fois un Groupe de travail chargé d'étudier les problèmes posés par l'adoption d'enfants étrangers. Il réunit des représentants de tous les ministères concernés : Justice, Affaires étrangères, Santé publique et d'autres services et associations ${ }^{45}$. Les nombreux rapports successifs montrent que la France a participé à toutes les discussions internationales sur cette question. En 1995, le Premier ministre Edouard Balladur écrivait au député Jean-François Mattéi : «il semble que les procédures administratives régissant l'adoption, et notamment l'adoption internationale, ne soient ni adaptées aux désirs des parents ni forcément respectueuses des Droits de l'Enfant ». D'où une mission confiée au député, qui doit être menée « en liaison avec le ministre d'Etat, Garde des Sceaux, le ministre d'Etat, ministre des Affaires sociales, de la Santé et de la Ville, le ministre des Affaires étrangères, le ministre délégué à l'Action humanitaire et aux Droits de l'Homme et le ministre délégué aux Affaires européennes » ${ }^{46}$. Cette énumération montre bien que le «dossier» adoption internationale est éminemment interministériel et concerne de grands portefeuilles dits régaliens. En 2000, suit le rapport Gouzes intitulé "Pour une éthique de l'adoption internationale ». De ces travaux émergent des réformes, des lois, des circulaires successives, d'où une complexité certaine pour saisir les compétences des différentes institutions successivement créées : Conseil Supérieur de l'Adoption (1975), Mission pour l'Adoption Internationale (1988), Autorité Centrale pour l'Adoption Internationale (1998), Agence Française pour l'Adoption (2005)...

Il faut ajouter que parmi les pays d'accueil, les analyses divergent sur l'approche juridique de l'adoption internationale. Alors que les pays de common law privilégient une approche juridictionnelle (Grande-Bretagne), la France, pays de droit romano-germanique a plutôt une approche conflictuelle, comme l'Allemagne. Mais celle-ci n'a pas la même histoire de l'adoption internationale que la France, sans doute en raison de la reconnaissance du droit du sang et non celui du sol dans ce pays. D'où de réelles difficultés quand il s'agit d'harmoniser les attitudes des Etats européens sur l'adoption internationale ${ }^{47}$ ou de définir une position commune du Parlement Européen sur la difficile question de l'adoption en Roumanie $^{48}$.

\section{La logique universelle des droits et du bien-être de l'enfant}

Dès l'entre-deux-guerres, se créèrent des organisations internationales dont le but était d'affirmer et de garantir les droits des enfants. En 1923, Eglantyne Jebb rédigea une

\footnotetext{
${ }^{45}$ Voir Yves Denéchère, «La diplomatie française face à la nouveauté des adoptions internationales d'enfants (années 1960 et 1970) », in Revue d'Histoire Diplomatique, parution en 2009.

${ }^{46}$ Jean-François Mattéi, Enfant d'ici, enfant d'ailleurs. L'adoption sans frontière, La Documentation Française, 1995. Lettre de mission d'Edouard Balladur, p.3.

${ }^{47}$ Voir Amélie Demange, L'adoption : un défi potentiel pour une Union européenne en mutation, Publications de l'Université Jean Moulin, 2005, 137 p.

${ }^{48}$ Cette question met également en lumière l'action de personnalités singulières qui se sont investies sur le dossier de l'adoption comme la baronne Emma Nicholson.
} 
déclaration des droits de l'enfance et la fit adopter par l'UISE comme sa charte fondamentale. En 1924, le Service Social International fut créé à son tour. En France, la loi de 1923 posa comme principe de base que l'adoption d'un enfant a pour but de lui trouver une famille et non plus d'assurer une descendance à une famille (comme dans le code civil de 1804) ; le mouvement de la prise en compte de l'intérêt supérieur et prépondérant de l'enfant était en marche. Après la Seconde Guerre mondiale, l'UNICEF (créée en 1946) a d'abord eu pour but de soutenir les enfants face aux misères de sortie de guerre, jamais elle ne s'occupa d'adoption. En 1959, la Déclaration des Droits de l'Enfant en 10 points constitua une véritable reconnaissance des droits des enfants au niveau international.

Sur «l'adoption entre pays », un premier rapport d'un groupe d'experts européens paraît en $1958^{49}$. Deux ans plus tard, un cycle d'études est organisé à Leysin (Suisse) sous l'égide de l'ONU. Etait déjà mentionnée la nécessité d'envisager les autres possibilités existant pour venir en aide aux enfants dans leur pays. La croissance du nombre des adoptions internationales et du nombre de pays concernés rendit nécessaire l'établissement de principes communs, d'où la convention de La Haye en matière de juridiction, loi applicable et reconnaissance des jugements d'adoption en 1965. En 1967, la Convention européenne en matière d'adoption des enfants du Conseil de l'Europe - dont le Comité social travaillait sur cette question depuis le début des années 1960 - fut le résultat d'une réflexion régionale plus qu'universelle qui fit la part belle aux lois des adoptants. La Convention de La Paz de 1984 qui privilégie les lois des adoptés, en fut en quelque sorte une réponse ${ }^{50}$. L'histoire de ces premières mobilisations, de la diplomatie mise en œuvre, des résistances des uns et de la détermination des autres est à faire. Pour quelles raisons la France n'a-t-elle pas signé les premières conventions internationales des années 1960 ? Les travaux préparatoires des trois grands textes postérieurs et de portée véritablement universelle des années 1980 et 1990 sont mieux connus et la documentation plus accessible (Déclaration des Nations Unies de 1986, Convention des Nations Unies sur les Droits de l'Enfant de 1989 et Convention de La Haye de 1993). Ces textes de référence placent au centre de toutes les adoptions internationales, l'intérêt supérieur de l'enfant, mais sans le définir explicitement tant il est difficile de concilier les positions des Etats.

Sans doute le passage d'une compétence relevant exclusivement des Etats à une pratique normée internationale - sinon universelle - peut être comparé avec la marche vers une justice internationale. Dans les deux cas, les Etats doivent accepter d'abandonner une partie de leur souveraineté pour qu'émerge un droit international réclamé par les ONG dans le cadre institutionnel international. Cependant, pas plus qu'au niveau des associations françaises, il n'y a eu de positions unanimes des ONG sur l'adoption internationale. Médecins du Monde, Mouvement Mondial des Mères et beaucoup d'autres ont défendu le principe d'une adoption internationale contrôlée et maîtrisée et devant être mise en œuvre après l'adoption dans le pays d'origine comme le préconise le Centre international de Référence pour les droits de l'enfant privé de famille. D'autres organisations ont exprimé une opposition active à l'adoption internationale. Régulièrement, l'UNICEF a dénoncé la dérive principale : quand le désir d'enfant de la part de candidats à l'adoption provoque l'abandon d'enfants par leurs familles biologiques. Dans les années 1980, l'ONG Défense des Enfants-International a multiplié les rapports sur les abus de l'adoption internationale en Amérique latine ${ }^{51}$. La fédération internationale de TDH poursuit ses initiatives pour convaincre gouvernements,

\footnotetext{
${ }^{49}$ AQO, CAAC, $\mathrm{n}^{\circ} 3$, Adoption entre pays. Rapport d'un groupe d'experts européens, Nations Unies, Genève, 1958, 58 p.

${ }^{50}$ Isabelle Cogliati, «Adoption internationale et respect de l'enfant », op. cit.

${ }^{51}$ Rapports de DEI sur la question du trafic et de la vente d'enfants en Bolivie (1987) et en Argentine (1989).
} 
institutions et $\mathrm{ONG}$ des dérives d'un système de relations Sud/Nord qui ne peut mener qu'à l'échec ${ }^{52}$.

Au-delà d'une actualité trop souvent sensationnelle et au regard des sources désormais disponibles, il est possible de faire l'histoire des différentes logiques interactives qui soustendent l'adoption internationale. Aujourd'hui, adoptants et adoptés, Etat et associations sont prêts à regarder en arrière pour mieux aller de l'avant. L'enjeu historique consiste à saisir une réalité complexe qui a certes produit des archives très confidentielles - et en ce sens l'enjeu se double d'une gageure - mais aussi de nombreuses archives administratives qui renseignent parfaitement sur les philosophies à l'œuvre, les argumentaires populationnistes, humanitaires et politiques, les mécanismes mis en route, les stratégies de secret élaborées. L'histoire de l'adoption internationale est donc possible, certes des limites sont identifiables mais le mouvement peut l'emporter.

Plus tard, lorsque ce chantier aura avancé pour la France, il sera intéressant de tenter une histoire comparée au regard d'autres pays d'accueil comme l'Italie ou l'Allemagne et de renverser l'angle d'approche en focalisant sur les pays-sources. On pourra également penser à mener d'autres recherches sur la situation des enfants étrangers isolés en France et sur d'autres questions comme par exemple les parrainages.

Si l'enjeu est historique, il est également mémoriel. «Et les enfants ? » pourra-t-on justement se demander en fin de lecture de cet article... Certes ils ont été les protagonistes le plus souvent passifs de l'histoire de l'adoption internationale, seuls les plus âgés d'entre eux, ont pu «choisir » leur famille d'accueil. Les enfants adoptés dans les années 1970 et 1980 sont devenus adultes. Demain, bien davantage qu'aujourd'hui, quand les cohortes nombreuses des adoptés des années 1990 et 2000 deviendront adultes à leur tour, ils seront de plus en plus acteurs de phénomènes internationaux induits par l'adoption internationale ${ }^{53}$ : recherche de leurs racines, aide au développement des pays d'origine, traits d'union culturels et économiques, etc. L'historien, qui ne saurait s'éloigner trop de l'humain, peut aussi leur apporter sa contribution.

Yves Denéchère

Professeur d'histoire contemporaine CERHIO UMR 6258 - Université d'Angers

\footnotetext{
52 «Campagne internationale contre le trafic d'enfants», et résultats de l'étude comparative « Adoption à quel prix ? Pour une responsabilité éthique des pays d'accueil dans l'adoption internationale » présentés en février 2008 au Parlement européen à Bruxelles.

${ }^{53}$ L'association «Racines Coréennes », fondée en 1995 par de jeunes Français d'origine coréenne (adoptés). publie la revue Hamkae (coréen, « Ensemble » en français).
} 